\title{
RELATIONSHIPS AMONG FOURIER-YEH-FEYNMAN TRANSFORM, CONVOLUTION AND THE FIRST VARIATION ON YEH-WIENER SPACE
}

\author{
Bong Jin Kim And Byoung Soo Kim
}

\begin{abstract}
We examine the various relationships that exist among the Fourier-Yeh-Feynman transform, convolution and the first variation for functionals on Yeh-Wiener space that belong to a Banach algebra $\mathcal{S}(Q)$.
\end{abstract}

\section{Definitions and preliminaries}

Let $Q=[0, S] \times[0, T]$ and let $C(Q)$ denote Yeh-Wiener space; that is, the space of all real-valued continuous functions $x(s, t)$ on $Q$ with $x(s, 0)=x(0, t)=0$ for all $0 \leq s \leq S$ and $0 \leq t \leq T$. Yeh [15] defined a Gaussian measure $m_{Y}$ on $C(Q)$ (later modified in [16]) such that as a stochastic process $\{x(s, t):(s, t) \in Q\}$ has mean $E[x(s, t)]=0$ and covariance $E[x(s, t) x(u, v)]=\min \{s, u\} \min \{t, v\}$.

Let $\mathcal{M}$ denote the class of all Yeh-Wiener measurable subsets of $C(Q)$ and we denote the Yeh-Wiener integral of a Yeh-Wiener integrable functional $F$ by

$$
\int_{C(Q)} F(x) d m_{Y}(x)
$$

A subset $E$ of $C(Q)$ is said to be scale-invariant measurable provided $\rho E$ is Yeh-Wiener measurable for every $\rho>0$, and a scale-invariant measurable set $N$ is said to be scale-invariant null provided $m_{Y}(\rho N)=0$ for every $\rho>0$. A property that holds except on a scale-invariant null set is said to hold scale-invariant almost everywhere ( $s$-a.e.). Given two complex-valued functions $F$ and $G$ on $C(Q)$, we say that $F=G$ s-a.e.

Received March 17, 2011. Accepted April 22, 2011.

2000 Mathematics Subject Classification. 28C20.

Key words and phrases. Yeh-Wiener space, Fourier-Yeh-Feynman transform, convolution, first variation.

Correspondence should be addressed to B.S. Kim, mathkbs@seoultech.ac.kr 
and write $F \approx G$ if $F(\rho x)=G(\rho x)$ for $m_{Y}$ almost every $x \in C(Q)$ for all $\rho>0$.

Let $\mathbb{C}_{+}$and $\mathbb{C}_{+}^{\sim}$ denote the set of complex numbers with positive real part and nonzero complex numbers with nonnegative real part, respectively.

Let $F$ be a complex valued measurable functional on $C(Q)$ such that

$$
J_{F}(\lambda)=\int_{C(Q)} F\left(\lambda^{-1 / 2} x\right) d m_{Y}(x)
$$

exists as a finite number for all real $\lambda>0$. If there exists a function $J_{F}^{*}(\lambda)$ analytic in $\mathbb{C}_{+}$such that $J_{F}^{*}(\lambda)=J_{F}(\lambda)$ for all $\lambda>0$, then $J_{F}^{*}(\lambda)$ is defined to be the analytic Yeh-Wiener integral of $F$ over $C(Q)$ with parameter $\lambda$, and for $\lambda \in \mathbb{C}_{+}$we write

$$
\int_{C(Q)}^{\mathrm{ayw}_{\lambda}} F(x) d m_{Y}(x)=J_{F}^{*}(\lambda) .
$$

If the following limit exists for nonzero real $q$, then we call it the analytic Yeh-Feynman integral of $F$ over $C(Q)$ with parameter $q$ and we write

$$
\int_{C(Q)}^{\mathrm{ayf}_{q}} F(x) d m_{Y}(x)=\lim _{\lambda \rightarrow-i q} \int_{C(Q)}^{\mathrm{ayw}_{\lambda}} F(x) d m_{Y}(x)
$$

where $\lambda$ approaches $-i q$ through $\mathbb{C}_{+}$.

Now we introduce the definitions of analytic Fourier-Yeh-Feynman transform, convolution and the first variation for functionals defined on $C(Q)$. Let $q$ be a nonzero real number.

Definition 1.1. Let $F$ be a function on $C(Q)$. For $\lambda \in \mathbb{C}_{+}$and $y \in C(Q)$, let

$$
T_{\lambda}(F)(y)=\int_{C(Q)}^{\mathrm{ayw}_{\lambda}} F(x+y) d m_{Y}(x) .
$$

For $1<p<\infty$, we define the $L_{p}$ analytic Fourier-Yeh-Feynman transform $T_{q}^{(p)}(F)$ of $F$ on $C(Q)$ by the formula $\left(\lambda \in \mathbb{C}_{+}\right)$

$$
T_{q}^{(p)}(F)(y)=\underset{\lambda \rightarrow-i q}{\operatorname{li} \operatorname{mos}_{\lambda}} T_{\lambda}(F)(y),
$$

whenever this limit exists; that is, for each $\rho>0$,

$$
\lim _{\lambda \rightarrow-i q} \int_{C(Q)}\left|T_{\lambda}(F)(\rho x)-T_{q}^{(p)}(F)(\rho x)\right|^{p^{\prime}} d m_{Y}(x)=0
$$


where $1 / p+1 / p^{\prime}=1$. We define the $L_{1}$ analytic Fourier-Yeh-Feynman transform $T_{q}^{(1)}(F)$ of $F$ by $\left(\lambda \in \mathbb{C}_{+}\right)$

$$
T_{q}^{(1)}(F)(y)=\lim _{\lambda \rightarrow-i q} T_{\lambda}(F)(y),
$$

for s-a.e. $y \in C(Q)$, whenever this limit exists [12].

Recently, Kim, Kim and Yoo [10, 11] studied an integral transform which is different from the Fourier-Yeh-Feynman transform on YehWiener space.

Definition 1.2. Let $F$ and $G$ be functionals on $C(Q)$. For $\lambda \in \mathbb{C}_{+}$, we define their convolution by

$$
(F * G)_{\lambda}(y)=\int_{C(Q)}^{\mathrm{ayw}_{\lambda}} F\left(\frac{y+x}{\sqrt{2}}\right) G\left(\frac{y-x}{\sqrt{2}}\right) d m_{Y}(x)
$$

if it exists. Moreover if $\lambda=-i q$ for nonzero real $q$, the convolution is defined by

$$
(F * G)_{q}(y)=\int_{C(Q)}^{\operatorname{ayf}_{q}} F\left(\frac{y+x}{\sqrt{2}}\right) G\left(\frac{y-x}{\sqrt{2}}\right) d m_{Y}(x)
$$

if it exists [12].

It is easy to see that commutative law and distributive law hold for the convolution.

Definition 1.3. Let $F$ be a function on $C(Q)$ and let $w \in C(Q)$. We define the first variation of $F(x)$ in the direction $w$ by

$$
\delta F(x \mid w)=\left.\frac{\partial}{\partial t} F(x+t w)\right|_{t=0}
$$

if it exists.

Various results involving Fourier-Feynman transform on Wiener space have been established and research based on this definition is continuing at the present time. For a detailed survey of the previous work on the Fourier-Feynman transform and related topics, see [14].

Next we describe the class of functionals that we work with in this paper after which we will describe the results of this paper.

The Banach algebra $\mathcal{S}(Q)$ consists of functionals expressible in the form

$$
F(x)=\int_{L_{2}(Q)} \exp \{i\langle\alpha, x\rangle\} d f(\alpha)
$$


for $s$-a.e. $x$ in $C(Q)$, where $f$ is an element of $M\left(L_{2}(Q)\right)$, the space of complex valued countably additive Borel measures on $L_{2}(Q)$ and $\langle\alpha, x\rangle$ denotes the Paley-Wiener-Zygmund stochastic integral $\int_{Q} \alpha(s, t) d x(s, t)$. Note that, for $\alpha \in L_{2}(Q)$, the PWZ integral $\langle\alpha, x\rangle$ exists for $s$-a.e. $x \in C(Q)$.

The Banach algebra $\mathcal{S}(Q)$ is the Yeh-Wiener space version of the Banach algebra $\mathcal{S}$ on classical Wiener space introduced by Cameron and Storvick in [3].

It is known that

$$
\int_{C(Q)} \exp \{i\langle\alpha, x\rangle\} d m_{Y}(x)=\exp \left\{-\frac{1}{2}\|\alpha\|^{2}\right\}
$$

and

$$
\int_{C(Q)}|\langle\alpha, x\rangle| d m_{Y}(x)=\left(\frac{2}{\pi}\right)^{1 / 2}\|\alpha\|,
$$

where $\|\alpha\|=\left(\int_{Q}(\alpha(s, t))^{2} d s d t\right)^{1 / 2}$. Moreover if $F \in \mathcal{S}(Q)$ is given by (1.12), then $F$ is analytic Yeh-Feynman integrable and

$$
\int_{C(Q)}^{\mathrm{ayf}_{q}} F(x) d m_{Y}(x)=\int_{L_{2}(Q)} \exp \left\{-\frac{i}{2 q}\|\alpha\|^{2}\right\} d f(\alpha) .
$$

We are now ready to discuss the results of this paper. Kim and Yang [12] established existence of Fourier-Yeh-Feynman transform and convolution of functionals in the Banach algebra $\mathcal{S}(Q)$ on Yeh-Wiener space. We restate the results in Section 2 of this paper. Moreover we will show that the first variation of functionals in $\mathcal{S}(Q)$ exists and also belongs to $\mathcal{S}(Q)$.

In Section 3, we examine all relationships involving exactly two of the three concepts of Fourier-Yeh-Feynman transform, convolution and the first variation of functionals in $\mathcal{S}(Q)$.

Finally in Section 4, we examine some of the relationships involving all three of these concepts where each concepts is used exactly once. The results in this paper extend previous results on Wiener space $[1,2,4,7$, $8,9,13]$ or abstract Wiener space $[5,6]$ to the Yeh-Wiener space.

\section{Transform, convolution and the first variation}

In [12], Kim and Yang obtained several results involving the FourierYeh-Feynman transform and convolution for functionals in $\mathcal{S}(Q)$. We 
begin this section by restating the existence of the Fourier-Yeh-Feynman transform and convolution for functionals in $\mathcal{S}(Q)$.

Theorem 2.1 (Theorem 3.1 in [12]). Let $F \in \mathcal{S}(Q)$ be given by (1.12). Then for all $p$ with $1 \leq p<\infty$ and for all nonzero real $q$, the Fourier-Yeh-Feynman transform $T_{q}^{(p)}(F)$ exists, belongs to $\mathcal{S}(Q)$ and is given by the formula

$$
T_{q}^{(p)}(F)(y)=\int_{L_{2}(Q)} \exp \{i\langle\alpha, y\rangle\} d f_{t}(\alpha)
$$

for s-a.e. $y$ in $C(Q)$, where $f_{t}$ is the measure defined by

$$
f_{t}(E)=\int_{E} \exp \left\{-\frac{i}{2 q}\|\alpha\|^{2}\right\} d f(\alpha)
$$

for $E \in \mathcal{B}\left(L_{2}(Q)\right)$.

Theorem 2.2 (Theorem 3.4 in [12]). Let $F, G \in \mathcal{S}(Q)$ with corresponding finite Borel measures $f$ and $g$ in $M\left(L_{2}(Q)\right)$, respectively. Then for all nonzero real $q$, the convolution product $(F * G)_{q}$ exists, belongs to $\mathcal{S}(Q)$ and is given by the formula

$$
(F * G)_{q}(y)=\int_{L_{2}(Q)} \exp \{i\langle\gamma, y\rangle\} d h_{c}(\gamma)
$$

for s-a.e. $y$ in $C(Q)$, where $h_{c}=h \circ \phi^{-1}$ and $\phi: L_{2}(Q)^{2} \rightarrow L_{2}(Q)$ is a function defined by $\phi(\alpha, \beta)=\frac{1}{\sqrt{2}}(\alpha+\beta)$ and $h$ is the measure defined by

$$
h(E)=\int_{E} \exp \left\{-\frac{i}{4 q}\|\alpha-\beta\|^{2}\right\} d f(\alpha) d g(\beta)
$$

for $E \in \mathcal{B}\left(L_{2}(Q)^{2}\right)$.

In the next theorem, we obtain a formula for the first variation of functionals in $\mathcal{S}(Q)$.

Theorem 2.3. Let $F \in \mathcal{S}(Q)$ be given by (1.12) and assume that $\|\cdot\|$ is $|f|$-integrable over $L_{2}(Q)$, that is, $\int_{L_{2}(Q)}\|\alpha\| d|f|(\alpha)<\infty$. Then the first variation $\delta F(x \mid w)$ exists, belongs to $\mathcal{S}(Q)$ as a function of $y$ and is given by the formula

$$
\delta F(y \mid w)=\int_{L_{2}(Q)} \exp \{i\langle\alpha, y\rangle\} d f_{v}(\alpha)
$$


for s-a.e. $y$ and $w$ in $C(Q)$, where $f_{v}$ is the measure in $M\left(L_{2}(Q)\right)$ defined by

$$
f_{v}(E)=\int_{E} i\langle\alpha, w\rangle d f(\alpha)
$$

for each $E \in \mathcal{B}\left(L_{2}(Q)\right)$.

Proof. Using (1.12), we have

$$
\delta F(y \mid w)=\left.\frac{\partial}{\partial t}\left(\int_{L_{2}(Q)} \exp \{i\langle\alpha, y\rangle+i t\langle\alpha, w\rangle\} d f(\alpha)\right)\right|_{t=0}
$$

for $s$-a.e. $y$ and $w$ in $C(Q)$. Now we obtain

$$
\delta F(y \mid w)=\int_{L_{2}(Q)} i\langle\alpha, w\rangle \exp \{i\langle\alpha, y\rangle\} d f(\alpha),
$$

provided we can justify interchanging the differentiation and the integral sign. It suffices to show that $\int_{L_{2}(Q)}|\langle\alpha, w\rangle| d|f|(\alpha)<\infty$ for $s$-a.e. $w$ in $C(Q)$. But by Fubini Theorem and the integration formula (1.14), we see that

$$
\int_{C(Q)} \int_{L_{2}(Q)}|\langle\alpha, w\rangle| d|f|(\alpha) d m_{Y}(w)=\left(\frac{2}{\pi}\right)^{1 / 2} \int_{L_{2}(Q)}\|\alpha\| d|f|(\alpha)
$$

which is finite by the assumption, and so $\int_{L_{2}(Q)}|\langle\alpha, w\rangle| d|f|(\alpha)<\infty$ for $s$-a.e. $w$ in $C(Q)$ as desired. Finally, it is easy to see that $\delta F(y \mid w)$ in (2.7) can be rewritten as (2.5) and (2.6) and this completes the proof.

Taking further the first variations, we obtain the following corollary.

Corollary 2.4. Let $n$ be a positive integer. Let $F \in \mathcal{S}(Q)$ be given by (1.12) and assume that $\|\cdot\|^{n}$ is $|f|$-integrable over $L_{2}(Q)$. Then

$$
\begin{aligned}
& \delta^{n} F\left(\cdot \mid w_{1}\right) \cdots\left(\cdot \mid w_{n-1}\right)\left(y \mid w_{n}\right) \\
= & \int_{L_{2}(Q)} i^{n}\left\langle\alpha, w_{1}\right\rangle \cdots\left\langle\alpha, w_{n}\right\rangle \exp \{i\langle\alpha, y\rangle\} d f(\alpha),
\end{aligned}
$$

for s-a.e. $y$ and $w_{1}, \ldots, w_{n}$ in $C(Q)$.

Proof. By induction, it suffices to take $n=2$ and prove that

$$
\delta^{2} F\left(\cdot \mid w_{1}\right)\left(y \mid w_{2}\right)=\int_{L_{2}(Q)} i^{2}\left\langle\alpha, w_{1}\right\rangle\left\langle\alpha, w_{2}\right\rangle \exp \{i\langle\alpha, y\rangle\} d f(\alpha),
$$


for $s$-a.e. $y$ and $w_{1}, w_{2}$ in $C(Q)$. Using (2.7), we have

$$
\begin{aligned}
\delta^{2} F\left(\cdot \mid w_{1}\right)\left(y \mid w_{2}\right) & =\left.\frac{\partial}{\partial t}\left(\delta F\left(y+t w_{2} \mid w_{1}\right)\right)\right|_{t=0} \\
& =\left.\frac{\partial}{\partial t}\left(\int_{L_{2}(Q)} i\left\langle\alpha, w_{1}\right\rangle \exp \left\{i\langle\alpha, y\rangle+i t\left\langle\alpha, w_{2}\right\rangle\right\} d f(\alpha)\right)\right|_{t=0} .
\end{aligned}
$$

Now the result follows if we can show that $\int_{L_{2}(Q)}\left|\left\langle\alpha, w_{1}\right\rangle\left\langle\alpha, w_{2}\right\rangle\right| d|f|(\alpha)$ is finite for $s$-a.e. $w_{1}, w_{2}$ in $C(Q)$. But by Fubini Theorem and the Yeh-Wiener integration formula, we see that

$$
\begin{aligned}
& \int_{C(Q)^{2}} \int_{L_{2}(Q)}\left|\left\langle\alpha, w_{1}\right\rangle\left\langle\alpha, w_{2}\right\rangle\right| d|f|(\alpha) d m_{Y}^{2}\left(w_{1}, w_{2}\right) \\
= & \frac{2}{\pi} \int_{L_{2}(Q)}\|\alpha\|^{2} d|f|(\alpha)
\end{aligned}
$$

which is finite by the assumption, and this completes the proof.

\section{Relationships involving two concepts}

In this section we establish various relationships involving exactly two of the concepts the Fourier-Yeh-Feynman transform, the convolution and the first variation of functionals in the Banach algebra $\mathcal{S}(Q)$ where each operation is used only once. In view of Theorems 2.1 through 2.3 in Section 2 above, all of the functionals in this section are elements of $\mathcal{S}(Q)$ as a function of $y$.

We begin this section with two theorems relating Fourier-Yeh-Feynman transform and convolution. In our first theorem, we restate a result in [12].

Theorem 3.1 (Theorem 3.6 in [12]). Let $F, G \in \mathcal{S}(Q)$ be given as in Theorem 2.2 and let $1 \leq p<\infty$. Then for all nonzero real $q$,

$$
T_{q}^{(p)}(F * G)_{q}(y)=T_{q}^{(p)}(F)\left(\frac{y}{\sqrt{2}}\right) T_{q}^{(p)}(G)\left(\frac{y}{\sqrt{2}}\right)
$$

for s-a.e. $y$ in $C(Q)$. Also both of the above expressions are given by the formula

$$
\int_{L_{2}(Q)^{2}} \exp \left\{\frac{i}{\sqrt{2}}\langle\alpha+\beta, y\rangle-\frac{i}{2 q}\|\alpha\|^{2}-\frac{i}{2 q}\|\beta\|^{2}\right\} d f(\alpha) d g(\beta) .
$$

Theorem 3.2. Let F, G, $p$ and $q$ be given as in Theorem 3.1. Then

$$
\left(T_{q}^{(p)}(F) * T_{q}^{(p)}(G)\right)_{-q}(y)=T_{q}^{(p)}\left(F\left(\frac{\cdot}{\sqrt{2}}\right) G\left(\frac{\cdot}{\sqrt{2}}\right)\right)(y)
$$


for s-a.e. $y$ in $C(Q)$. Also, both of the expressions in (3.3) are given by the formula

$$
\int_{L_{2}(Q)^{2}} \exp \left\{\frac{i}{\sqrt{2}}\langle\alpha+\beta, y\rangle-\frac{i}{4 q}\|\alpha+\beta\|^{2}\right\} d f(\alpha) d g(\beta) .
$$

Proof. The transforms $T_{q}^{(p)}(F)$ and $T_{q}^{(p)}(G)$ are expressed as (2.1) with corresponding measures $f_{t}$ and $g_{t}$, where $g_{t}$ is given by (2.2) with $f$ replaced by $g$. Applying Theorem 2.2 to the expression (2.1) for $F$ and $G$, we obtain

$$
\left(T_{q}^{(p)}(F) * T_{q}^{(p)}(G)\right)_{-q}(y)=\int_{L_{2}(Q)} \exp \{i\langle\gamma, y\rangle\} d h_{c}(\gamma),
$$

where $h_{c}=h \circ \phi^{-1}$,

$$
h(E)=\int_{E} \exp \left\{\frac{i}{4 q}\|\alpha-\beta\|^{2}\right\} d f_{t}(\alpha) d g_{t}(\beta)
$$

for $E \in \mathcal{B}\left(L_{2}(Q)^{2}\right)$ and $\phi$ is given as in Theorem 2.2. By the expression (2.2) for $f_{t}$ and $g_{t}$, we obtain (3.4).

On the other hand, by (1.12),

$$
F\left(\frac{x}{\sqrt{2}}\right) G\left(\frac{x}{\sqrt{2}}\right)=\int_{L_{2}(Q)} \exp \{i\langle\gamma, x\rangle\} d k(\gamma),
$$

where $k=l \circ \phi^{-1}$ and $l(E)=\int_{E} d f(\alpha) d g(\beta)$ for $E \in \mathcal{B}\left(L_{2}(Q)^{2}\right)$. Hence by Theorem 2.1,

$$
T_{q}^{(p)}\left(F\left(\frac{\cdot}{\sqrt{2}}\right) G\left(\frac{\cdot}{\sqrt{2}}\right)\right)(y)=\int_{L_{2}(Q)} \exp \{i\langle\gamma, y\rangle\} d k_{t}(\gamma),
$$

where $k_{t}(E)=\int_{E} \exp \left\{-\frac{i}{2 q}\|\gamma\|^{2}\right\} d k(\gamma)$ for $E \in \mathcal{B}\left(L_{2}(Q)\right)$. Hence we know that the right hand side also expressed as (3.4), which completes the proof.

In the next two theorems, we obtain formulas involving Fourier-YehFeynman transform and the first variation of a functional $F$ in $\mathcal{S}(Q)$. In Theorem 3.3 below, we consider $\delta F(y \mid w)$ as a function of $y$, while in Theorem 3.5 below, we consider $\delta F(y \mid w)$ as a function of $w$.

Theorem 3.3. Let $F$ be in $\mathcal{S}(Q)$ be given by (1.12) and assume that $\|\cdot\|$ is $|f|$-integrable over $L_{2}(Q)$. Then for all $1 \leq p<\infty$ and all nonzero real $q$,

$$
T_{q}^{(p)}(\delta F(\cdot \mid w))(y)=\delta T_{q}^{(p)}(F)(y \mid w)
$$


for s-a.e. $y$ and $w$ in $C(Q)$. Also, both of the expressions in (3.5) are given by the formula

$$
\int_{L_{2}(Q)} i\langle\alpha, w\rangle \exp \left\{i\langle\alpha, y\rangle-\frac{i}{2 q}\|\alpha\|^{2}\right\} d f(\alpha)
$$

for s-a.e. $y$ and $w$ in $C(Q)$.

Proof. Note that $\delta F(y \mid w)$ is given by (2.5). By Theorem 2.1, we have

$$
T_{q}^{(p)}(\delta F(\cdot \mid w))(y)=\int_{L_{2}(Q)} \exp \{i\langle\alpha, y\rangle\} d\left(f_{v}\right)_{t}(\alpha)
$$

for $s$-a.e. $y$ and $w$ in $C(Q)$. By $(2.2)$ we have

$$
T_{q}^{(p)}(\delta F(\cdot \mid w))(y)=\int_{L_{2}(Q)} \exp \left\{i\langle\alpha, y\rangle-\frac{i}{2 q}\|\alpha\|^{2}\right\} d f_{v}(\alpha)
$$

and by (2.6), we have (3.6). On the other hand, by applying Theorem 2.3 to the expression $(2.1)$ for $T_{q}^{(p)}(F)$, we see that the right hand side of (3.5) is also expressed as (3.6).

Taking further variations of the expression given in (3.6), we obtain the following corollary.

Corollary 3.4. Let $n$ be a positive integer. Let $F \in \mathcal{S}(Q)$ be given by (1.12) and assume that $\|\cdot\|^{n}$ is $|f|$-integrable over $L_{2}(Q)$. Then for all $1 \leq p<\infty$ and all nonzero real $q$,

$$
T_{q}^{(p)}\left(\delta^{n} F\left(\cdot \mid w_{1}\right) \cdots\left(\cdot \mid w_{n}\right)\right)(y)=\delta^{n} T_{q}^{(p)}(F)\left(\cdot \mid w_{1}\right) \cdots\left(\cdot \mid w_{n-1}\right)\left(y \mid w_{n}\right)
$$

for s-a.e. $y$ and $w_{1}, \ldots, w_{n}$ in $C(Q)$. Also, both of the expressions in (3.7) are given by the formula

$$
\int_{L_{2}(Q)} i^{n}\left\langle\alpha, w_{1}\right\rangle \cdots\left\langle\alpha, w_{n}\right\rangle \exp \left\{i\langle\alpha, y\rangle-\frac{i}{2 q}\|\alpha\|^{2}\right\} d f(\alpha),
$$

for $s$-a.e. $y$ and $w_{1}, \ldots, w_{n}$ in $C(Q)$.

Theorem 3.5. Let F, $p$ and $q$ be given as in Theorem 3.3. Then

$$
T_{q}^{(p)}(\delta F(y \mid \cdot))(w)=\delta F(y \mid w)
$$

for s-a.e. $y$ and $w$ in $C(Q)$. 
Proof. For $\lambda>0$, using the expression (2.7), we obtain

$$
\begin{aligned}
& T_{\lambda}(\delta F(y \mid \cdot))(w) \\
= & \int_{C(Q)} \delta F\left(y \mid \lambda^{-1 / 2} x+w\right) d m_{Y}(x) \\
= & \int_{C(Q)} \int_{L_{2}(Q)} i\left\langle\alpha, \lambda^{-1 / 2} x+w\right\rangle \exp \{i\langle\alpha, y\rangle\} d f(\alpha) d m_{Y}(x)
\end{aligned}
$$

for $s$-a.e. $y$ and $w$ in $C(Q)$. Since $\int_{C(Q)}\langle\alpha, x\rangle d m_{Y}(x)=0$, Fubini theorem enable us to conclude that

$$
T_{q}^{(p)}(\delta F(y \mid \cdot))(w)=\int_{L_{2}(Q)} i\langle\alpha, w\rangle \exp \{i\langle\alpha, y\rangle\} d f(\alpha) .
$$

By Theorem 2.3 we have (3.9).

In our next theorem we obtain a formula for the first variation of the convolution.

Theorem 3.6. Let $F$ and $G$ be elements of $\mathcal{S}(Q)$ with corresponding measures $f$ and $g$ in $M\left(L_{2}(Q)\right)$, respectively. Further, assume that $\|\cdot\|$ is $|f|$ and $|g|$-integrable over $L_{2}(Q)$. Then for all nonzero real $q$

$$
\begin{aligned}
\delta(F * G)_{q}(y \mid w)= & \frac{i}{\sqrt{2}} \int_{L_{2}(Q)^{2}}\langle\alpha+\beta, w\rangle \exp \left\{\frac{i}{\sqrt{2}}\langle\alpha+\beta, y\rangle\right. \\
& \left.-\frac{i}{4 q}\|\alpha-\beta\|^{2}\right\} d f(\alpha) d g(\beta)
\end{aligned}
$$

for s-a.e. $y$ and $w$ in $C(Q)$.

Proof. Applying Theorem 2.3 to the expression (2.3) we obtain (3.10).

Next we obtain formulas involving convolution and the first variation. In Theorem 3.7 below, we take the convolution with respect to the first argument of the variation, while in Theorem 3.8 below, we take the convolution with respect to the second argument of the variation.

Theorem 3.7. Let $F, G$ and $q$ be given as in Theorem 3.6. Then

$$
\begin{aligned}
& (\delta F(\cdot \mid w) * \delta G(\cdot \mid w))_{q}(y) \\
= & -\int_{L_{2}(Q)^{2}}\langle\alpha, w\rangle\langle\beta, w\rangle \exp \left\{\frac{i}{\sqrt{2}}\langle\alpha+\beta, y\rangle-\frac{i}{4 q}\|\alpha-\beta\|^{2}\right\} d f(\alpha) d g(\beta)
\end{aligned}
$$

for $s$-a.e. $y$ and $w$ in $C(Q)$. 
Proof. Applying Theorem 2.2 to the expression (2.5) for $F$ and $G$, we have the result.

Theorem 3.8. Let $F, G$ and $g$ be given as in Theorem 3.6. Then

$$
\begin{aligned}
& (\delta F(y \mid \cdot) * \delta G(y \mid \cdot))_{q}(w) \\
= & \delta F(y \mid w / \sqrt{2}) \delta G(y \mid w / \sqrt{2}) \\
& +\frac{i}{2 q} \int_{L_{2}(Q)^{2}}(\alpha, \beta) \exp \{i\langle\alpha+\beta, y\rangle\} d f(\alpha) d g(\beta)
\end{aligned}
$$

for s-a.e. $y$ and $w$ in $C(Q)$, where $(\alpha, \beta)$ denotes the inner product $\int_{Q} \alpha(s, t) \beta(s, t) d s d t$. Also, both of the expressions in (3.12) are given by the formula

$$
\text { (3.13) }-\frac{1}{2} \int_{L_{2}(Q)^{2}}\left[\langle\alpha, w\rangle\langle\beta, w\rangle-\frac{i}{q}(\alpha, \beta)\right] \exp \{i\langle\alpha+\beta, y\rangle\} d f(\alpha) d g(\beta) .
$$

Proof. Using the definition of the convolution, the equation (2.7) for $F$ and $G$, we see that for $\lambda>0$

$$
\begin{aligned}
& (\delta F(y \mid \cdot) * \delta G(y \mid \cdot))_{\lambda}(w) \\
= & \int_{C(Q)} \delta F\left(y \mid \frac{w+\lambda^{-1 / 2} x}{\sqrt{2}}\right) \delta G\left(y \mid \frac{w-\lambda^{-1 / 2} x}{\sqrt{2}}\right) d m_{Y}(x) \\
= & -\frac{1}{2} \int_{C(Q)} \int_{L_{2}(Q)^{2}}\left(\langle\alpha, w\rangle+\lambda^{-1 / 2}\langle\alpha, x\rangle\right)\left(\langle\beta, w\rangle-\lambda^{-1 / 2}\langle\beta, x\rangle\right) \\
& \cdot \exp \{i\langle\alpha+\beta, y\rangle\} d f(\alpha) d g(\beta) d m_{Y}(x)
\end{aligned}
$$

for $s$-a.e. $y$ and $w$ in $C(Q)$. But since $\int_{C(Q)}\langle\alpha, x\rangle d m_{Y}(x)=0$ and

$$
\int_{C(Q)}\langle\alpha, x\rangle\langle\beta, x\rangle d m_{Y}(x)=(\alpha, \beta),
$$

we have

$$
\begin{aligned}
& (\delta F(y \mid \cdot) * \delta G(y \mid \cdot))_{\lambda}(w) \\
= & -\frac{1}{2} \int_{L_{2}(Q)^{2}}\left[\langle\alpha, w\rangle\langle\beta, w\rangle-\frac{1}{\lambda}(\alpha, \beta)\right] \exp \{i\langle\alpha+\beta, y\rangle\} d f(\alpha) d g(\beta)
\end{aligned}
$$

by the Fubini theorem. Using Morera theorem, we can show that the last expression has an analytic extension on $\mathbb{C}_{+}$as a function of $\lambda$. Letting $\lambda \rightarrow-i q$, we have expression (3.13). By (2.7), we obtain (3.12). 


\section{Relationships involving all three concepts}

In this section, we look at some of the relationships involving the Fourier-Yeh-Feynman transform, the convolution and the first variation where each operation is used exactly once. There are more than six possibilities since one can take the Fourier-Yeh-Feynman transform or the convolution with respect to the first or second argument of the first variation. It turns out that there are 13 distinct formulas. Some of these are given by equations (4.1) through (4.6). We omit the details of the calculations used to obtain these equations because the techniques needed are similar to those used above in Section 3. Of course the remaining 7 formulas also can be obtained, and they are similar to the formulas in Section 4 of $[4,13]$.

It is interesting to note that the left hand side of each formula (4.1) through (4.6) involves all of the three operations, while the right hand sides involve one or two of the three operations.

Throughout this section, let $F$ and $G$ be elements of $\mathcal{S}(Q)$ with corresponding measures $f$ and $g$ in $M\left(L_{2}(Q)\right)$, respectively. Further, assume that $\|\cdot\|$ is $|f|$ and $|g|$-integrable over $L_{2}(Q)$. Let $1 \leq p<\infty$ and let $q$ be a nonzero real number.

We begin with formulas for the first variation of the Fourier-YehFeynman transform of the convolution.

Formula 4.1. Taking the first variation of the expression in (3.1) yields the formula

$$
\begin{aligned}
\left.\delta\left(T_{q}^{(p)}(F * G)_{q}\right)(y \mid w)\right)= & T_{q}^{(p)}(F)\left(\frac{y}{\sqrt{2}}\right) \delta T_{q}^{(p)}(G)\left(\frac{y}{\sqrt{2}} \mid \frac{w}{\sqrt{2}}\right) \\
& +\delta T_{q}^{(p)}(F)\left(\frac{y}{\sqrt{2}} \mid \frac{w}{\sqrt{2}}\right) T_{q}^{(p)}(G)\left(\frac{y}{\sqrt{2}}\right)
\end{aligned}
$$

for $s$-a.e. $y$ and $w$ in $C(Q)$.

In Formula 4.2 below, we take the Fourier-Yeh-Feynman transform with respect to the first argument of the first variation of the convolution, while in Formula 4.3 below, we take the Fourier-Yeh-Feynman transform with respect to the second argument of the first variation of the convolution. 
Formula 4.2. Applying Theorem 3.3 to $(F * G)_{q}$ and Formula 4.1 yields the formula

$$
\begin{aligned}
& T_{q}^{(p)}\left(\delta(F * G)_{q}(\cdot \mid w)\right)(y) \\
= & T_{q}^{(p)}(F)\left(\frac{y}{\sqrt{2}}\right) \delta T_{q}^{(p)}(G)\left(\frac{y}{\sqrt{2}} \mid \frac{w}{\sqrt{2}}\right)+\delta T_{q}^{(p)}(F)\left(\frac{y}{\sqrt{2}} \mid \frac{w}{\sqrt{2}}\right) T_{q}^{(p)}(G)\left(\frac{y}{\sqrt{2}}\right)
\end{aligned}
$$

for $s$-a.e. $y$ and $w$ in $C(Q)$.

Formula 4.3. Applying Theorem 3.5 to $(F * G)_{q}$ yields the formula

$$
T_{q}^{(p)}\left(\delta(F * G)_{q}(y \mid \cdot)\right)(w)=\delta(F * G)_{q}(y \mid w)
$$

for $s$-a.e. $y$ and $w$ in $C(Q)$.

In the following Formula 4.4, we obtain formulas for the Fourier-YehFeynman transform of $(\delta F(\cdot \mid w) * \delta G(\cdot \mid w))_{q}(y)$ with respect to $y$.

Formula 4.4. Applying Theorems 3.1 and 3.3 yields the formula

$$
\begin{aligned}
& T_{q}^{(p)}\left((\delta F(\cdot \mid w) * \delta G(\cdot \mid w))_{q}\right)(y) \\
= & T_{q}^{(p)}(\delta F(\cdot \mid w))\left(\frac{y}{\sqrt{2}}\right) T_{q}^{(p)}(\delta G(\cdot \mid w))\left(\frac{y}{\sqrt{2}}\right) \\
= & \delta T_{q}^{(p)}(F)\left(\frac{y}{\sqrt{2}} \mid w\right) \delta T_{q}^{(p)}(G)\left(\frac{y}{\sqrt{2}} \mid w\right)
\end{aligned}
$$

for $s$-a.e. $y$ and $w$ in $C(Q)$.

In the following Formula 4.5, we obtain formulas for the Fourier-YehFeynman transform of $(\delta F(y \mid \cdot) * \delta G(y \mid \cdot))_{q}(w)$ with respect to $w$.

Formula 4.5. Applying Theorems 3.1 and 3.5 yields the formula

$$
\begin{aligned}
& T_{q}^{(p)}\left((\delta F(y \mid \cdot) * \delta G(y \mid \cdot))_{q}\right)(w) \\
= & T_{q}^{(p)}(\delta F(y \mid \cdot))\left(\frac{w}{\sqrt{2}}\right) T_{q}^{(p)}(\delta G(y \mid \cdot))\left(\frac{w}{\sqrt{2}}\right) \\
= & \delta F\left(y \mid \frac{w}{\sqrt{2}}\right) \delta G\left(y \mid \frac{w}{\sqrt{2}}\right)
\end{aligned}
$$

for $s$-a.e. $y$ and $w$ in $C(Q)$.

In the following Formula 4.6, we obtain formula for the convolution with respect to the first argument of the first variation of the FourierYeh-Feynman transform. 
Formula 4.6. Applying Theorems 3.2 and 3.3 yields the formula

$$
\begin{aligned}
& \left(\delta T_{q}^{(p)}(F)(\cdot \mid w) * \delta T_{q}^{(p)}(G)(\cdot \mid w)\right)_{-q}(y) \\
= & \left(T_{q}^{(p)}(\delta F(\cdot \mid w)) * T_{q}^{(p)}(\delta G(\cdot \mid w))\right)_{-q}(y) \\
= & T_{q}^{(p)}\left(\delta F\left(\frac{\cdot}{\sqrt{2}} \mid w\right) \delta G\left(\frac{\cdot}{\sqrt{2}} \mid w\right)\right)(y)
\end{aligned}
$$

for $s$-a.e. $y$ and $w$ in $C(Q)$.

\section{References}

[1] J.M. Ahn, K.S. Chang, B.S. Kim and I. Yoo, Fourier-Feynman transform, convolution and first variation, Acta Math. Hungar. 100 (2003), 215-235.

[2] R.H. Cameron and D.A. Storvick, An $L_{2}$ analytic Fourier-Feynman transform, Michigan Math. J. 23 (1976), 1-30.

[3] _ Some Banach algebras of analytic Feynman integrable functionals, Analytic Functions (Kozubnik, 1979), Lecture Notes in Math. 798, Springer-Verlag, Berlin, 1980, 18-67.

[4] K.S. Chang, D.H. Cho, B.S. Kim, T.S. Song and I. Yoo, Relationships involving generalized Fourier-Feynman transform, convolution and first variation, Integral Transforms Spec. Funct. 16 (2005), 391-405.

[5] K.S. Chang, B.S. Kim and I. Yoo, Fourier-Feynman transform, convolution and first variation of functionals on abstract Wiener space, Integral Transforms Spec. Funct. 10 (2000), 179-200.

[6] Analytic Fourier-Feynman transform and convolution of functionals on abstract Wiener space, Rocky Mountain J. Math. 30 (2000), 823-842.

[7] T. Huffman, C. Park and D. Skoug, Analytic Fourier-Feynman transforms and convolution, Trans. Amer. Math. Soc. 347 (1995), 661-673.

[8] Convolutions and Fourier-Feynman transforms of functionals involving multiple integrals, Michigan Math. J. 43 (1996), 247-261.

[9] G.W. Johnson and D.L. Skoug, An $L_{p}$ analytic Fourier-Feynman transform, Michigan Math. J. 26 (1979), 103-127.

[10] B.J. Kim, B.S. Kim and I. Yoo, Integral transforms of functionals on a function space of two variables, J. Chungcheong Math. Soc. 23 (2010), 349-362.

[11] B.S. Kim, Integral transforms of square integrable functionals on Yeh-Wiener space, Kyungpook Math. J. 49 (2009), 155-166.

[12] B.S. Kim and Y.K. Yang, Fourier-Yeh-Feynman transform and convolution on Yeh-Wiener space, Korean J. Math. 16 (2008), 335-348.

[13] C. Park, D. Skoug and D. Storvick, Relationships among the first variation, the convolution product, and the Fourier-Feynman transform, Rocky Mountain J. Math. 28 (1998), 1447-1468.

[14] D. Skoug and D. Storvick, A survey of results involving transforms and convolutions in function space, Rocky Mountain J. Math. 34 (2004), 1147-1175.

[15] J. Yeh, Wiener measure in a space of functions of two variables, Trans. Amer. Math. Soc. 95 (1960), 433-450.

[16] Stochastic processes and the Wiener integral, Marcel Dekker, New York, 1973. 
Bong Jin Kim

Department of Mathematics, Daejin University,

Pocheon 487-711, Korea.

E-mail: bjkim@daejin.ac.kr

\section{Byoung Soo Kim}

School of Liberal Arts, Seoul National University of Science and Technology,

Seoul 139-743, Korea.

E-mail: mathkbs@seoultech.ac.kr 